\title{
Ethnicity, sex, and the incidence of congenital heart defects: a report from the National Down Syndrome Project
}

Sallie B. Freeman, $P h D^{1}$, Lora H. Bean, $P h D^{1}$, Emily G. Allen, $P h D^{1}$, Stuart W. Tinker, $B S^{1}$, Adam E. Locke, $B A^{1}$, Charlotte Druschel, MD, MPH', Charlotte A. Hobbs, MD, PhD ${ }^{3}$, Paul A. Romitti, PhD ${ }^{4}$, Marjorie H. Royle, PhD ${ }^{5}$, Claudine P. Torfs, $P h D^{6}$, Kenneth J. Dooley, $M D^{7}$, and Stephanie L. Sherman, $P h D^{1}$

\begin{abstract}
Purpose: The population-based National Down Syndrome Project combined epidemiological and molecular methods to study congenital heart defects in Down syndrome. Methods: Between 2000 and 2004, six sites collected DNA, clinical, and epidemiological information on parents and infants. We used logistic regression to examine factors associated with the most common Down syndrome-associated heart defects. Results: Of 1469 eligible infants, major cardiac defects were present in $44 \%$; atrioventricular septal defect (39\%), secundum atrial septal defect (42\%), ventricular septal defect (43\%), and tetralogy of Fallot (6\%). Atrioventricular septal defects showed the most significant sex and ethnic differences with twice as many affected females (odds ratio, 1.93; 95\% confidence interval, 1.40-2.67) and, compared with whites, twice as many blacks (odds ratio, 2.06; 95\% confidence interval, 1.32-3.21) and half as many Hispanics (odds ratio, 0.48; 95\% confidence interval, 0.300.77). No associations were found with origin of the nondisjunction error or with the presence of gastrointestinal defects. Conclusions: Sex and ethnic differences exist for atrioventricular septal defects in Down syndrome. Identification of genetic and environmental risk factors associated with these differences is essential to our understanding of the etiology of congenital heart defects. Genet Med 2008:10(3):173-180.
\end{abstract}

Key Words: Down syndrome, trisomy 21, congenital heart defects, atrioventricular septal defect, ethnicity, race, sex, gender, maternal age, ancestral informative markers

The National Down Syndrome Project (NDSP) seeks to investigate the etiology and phenotypic consequences of trisomy 21 Down syndrome (DS). ${ }^{1}$ Aside from the universal findings of mental retardation and hypotonia, congenital heart defects (CHDs) are arguably the most important clinical sequelae of an extra chromosome 21. In 1998 the Atlanta Down syndrome Project (ADSP), a forerunner of the NDSP, reported that $41 \%$ of newborns with DS were born with one or more major heart defects, including atrioventricular septal defect (AVSD), secundum atrial septal defect (ASDII), ventricular septal defect (VSD), and tetralogy of Fallot (TOF). ${ }^{2}$ Findings from the

\footnotetext{
From the ${ }^{1}$ Department of Human Genetics, Emory University, Atlanta, Georgia; ${ }^{2}$ New York State Department of Health, Troy, New York; ${ }^{3}$ College of Medicine, Department of Pediatrics, University of Arkansas for Medical Sciences, Little Rock, Arkansas; ${ }^{4}$ Department of Epidemiology, College of Public Health, University of Iowa, Iowa City, Iowa; ${ }^{5}$ New Jersey Department of Health and Senior Services, Trenton, New Jersey; ${ }^{6}$ Public Health Institute, Birth Defects Studies, Emeryville, California; and ${ }^{7}$ Sibley Heart Center Cardiology, Children's Healthcare of Atlanta, Atlanta, Georgia.

Sallie B. Freeman, PhD, Department of Human Genetics, Emory University, 2165 North Decatur Rd, Decatur, GA 30033. E-mail: sfreeman@genetics.emory.edu.

Disclosure: The authors declare no conflict of interest.

Submitted for publication July 11, 2007.

Accepted for publication October 24, 2007.

DOI: 10.1097/GIM.0b013e3181634867
}

ADSP and other recent population-based studies of DS and $\mathrm{CHDs}^{2-5}$ are summarized in Table 1.

With the birth prevalence of major DS-associated CHDs well established by multiple studies using modern diagnostic methods, attention can now be directed toward understanding the etiology of these defects. Not only do infants with DS have a higher rate of CHDs than do infants without DS, but one defect, the AVSD, is particularly characteristic. To understand the etiology of CHDs in DS and of AVSD specifically, both genetic and environmental determinants must be explored. For example, several recent reports have suggested that the distribution of CHDs in DS varies by ethnicity (race/ethnicity), ${ }^{6-13}$ but most population-based studies have not had broad ethnic representation (Table 1). Drawing on our experience with the ADSP, we designed the multicenter NDSP to explore possible CHD risk factors singly and in combination. The NDSP is one of the largest population-based studies of CHDs in DS and the first to assemble clinical, demographic, and molecular data on a large, ethnically diverse sample of individuals with DS and their parents.

This report focuses on the relationships between DS-related CHDs and ethnicity, sex, maternal age, and origin of the chromosome error. Importantly, it is unique in presenting the first 
Table 1

Population-based studies of congenital heart defects in down syndrome

\begin{tabular}{|c|c|c|c|c|c|}
\hline & $\begin{array}{l}\text { Freeman et al. }{ }^{2} \text { and } \\
\text { unpublished }\end{array}$ & Kallen et al. ${ }^{3}$ & Stoll et al. ${ }^{4}$ & $\begin{array}{c}\text { Torfs and } \\
\text { Christianson }^{5}\end{array}$ & Current study \\
\hline Study location & Atlanta GA & France and Sweden ${ }^{a}$ & France & California & $\begin{array}{l}\text { Arkansas, California, Atlanta, } \\
\text { Iowa, New Jersey, New York }\end{array}$ \\
\hline Study period & 1989-1999 & $1976-1993$ & 1979-1996 & $1983-1993$ & $2000-2004$ \\
\hline Number of cases ${ }^{b}$ & 423 & 3694 & 398 & 2894 & 1469 \\
\hline Biological samples collected & yes & no & no & no & yes \\
\hline \multicolumn{6}{|l|}{ Cardiac information by: } \\
\hline Sex & yes & yes & no & no & yes \\
\hline Ethnicity & yes & no & no & no & yes \\
\hline \multirow[t]{3}{*}{ Ethnicities represented at $\geq 10 \%$} & white & NA & white & NA & white \\
\hline & black & & & & black \\
\hline & & & & & Hispanic \\
\hline \multirow[t]{2}{*}{$\%$ with CHDs } & $41 \%$ & $23 \%$ France & $46 \%$ & $56 \%$ & $44 \%$ \\
\hline & & $32 \%$ Sweden & & & \\
\hline \multirow[t]{2}{*}{ AVSD } & $47 \%^{c}$ & $43 \%$ France & $43 \%^{c}$ & $31 \%^{c}$ & $39 \%^{c}$ \\
\hline & & $42 \%$ Sweden & & & \\
\hline \multirow[t]{2}{*}{ ASDII } & $37 \%^{c}$ & 4\% France & NA & $11 \%^{c}$ & $42 \%^{c}$ \\
\hline & & $8 \%$ Sweden & & & \\
\hline \multirow[t]{2}{*}{ VSD } & $44 \%^{c}$ & 17\% France & $32 \%^{c}$ & $11 \%^{c}$ & $43 \%^{c}$ \\
\hline & & $20 \%$ Sweden & & & \\
\hline TOF & $7 \%^{c}$ & $\begin{array}{l}\text { 3\% France } \\
3 \% \text { Sweden }\end{array}$ & $3 \%^{c}$ & $4 \%^{c}$ & $6 \%^{c}$ \\
\hline
\end{tabular}

${ }^{a}$ Data from an Italian hospital-based registry included in their paper were excluded from this table.

${ }^{b}$ Live births with or without stillbirths depending on study.

${ }^{c}$ Among those with any heart defect.

NA, not available.

molecular evidence to support the finding of ethnic differences in the incidence of AVSD in DS.

\section{SUBJECTS AND METHODS}

\section{NDSP subjects}

Based at Emory University in Atlanta, GA, the NDSP enrolled families of infants with DS born between 2000 and 2004 at six sites across the country: the Atlanta five-county metropolitan area (GA), statewide in Arkansas (AR), Iowa (IA), and New Jersey (NJ), as well as selected geographic areas of California (CA) and New York (NY). Details of ascertainment and recruitment were recently reported. ${ }^{1}$ Each NDSP site was linked to a birth defects surveillance system, and all sites had extensive experience in enrolling families, collecting infant medical data, and completing parental questionnaires. All NDSP sites obtained institutional review board approvals and informed consent from participants.

The NDSP included live born infants with either standard trisomy 21 or mosaic trisomy 21 born during the study period to English- or Spanish-speaking mothers living in the designated geographic areas. Infants with DS due to a trans- location were excluded as were families whose infants died after birth and before study enrollment. For the current report of CHDs, we have further excluded infants with mosaic trisomy 21 as well as those with standard trisomy 21 plus another clinically relevant chromosome abnormality.

\section{Other subjects}

For the ADSP, infants with DS born in Atlanta from 1989 through 1999 were ascertained by study personnel at Emory University in cooperation with the Metropolitan Atlanta Congenital Defects Program of the Centers for Disease Control and Prevention. The methodology of that study has been described previously ${ }^{2}$ and is nearly identical to that of the NDSP. For the examination of ancestral informative markers (AIMs), we included additional self-reported black individuals with DS from an ongoing study of CHDs based at Emory University ${ }^{14}$ as well as from the Sibley Heart Center, Cardiology, Children's Health Care of Atlanta.

\section{Clinical information}

Sites abstracted infant records and entered the information onto a structured clinical form, which was then reviewed by a 
single clinically trained individual at Emory. The presence or absence of CHDs, the particular heart defect(s) diagnosed, and the date and method(s) of diagnosis were recorded for each infant. Congenital gastrointestinal defects were also reported. Every effort was made to compile medical information based on the most definitive diagnostic tests used in each case. We placed an emphasis on obtaining the best information possible to document the major heart defects seen in DS, namely, AVSD, VSD, ASDII, and TOF. Each occurrence of a heart defect was counted. For example, in an infant with both an ASDII and a VSD, both defects were recorded. However, a VSD which was part of TOF was not counted separately. Patent ductus ateriosus (PDA) and patent foramen ovale (PFO) were not tallied because these were not uniformly reported by all sites. In addition, we did not include the diagnosis of "PFO rule out ASD" (PFO/ASD) but limited our count of ASDs to those clearly described as an ASDII.

\section{Demographic information}

Trained study personnel completed detailed questionnaires with participating mothers, recording self-reported maternal age, ethnicity, and country of birth. In addition, for both participating and nonparticipating mothers, independent information regarding maternal age and ethnicity was available from birth records. Coding of ethnicity varied somewhat from site to site, but for this report we reduced the groups to (1) white non-Hispanic, (2) black non-Hispanic, (3) Hispanic, (4) American Indian/Alaskan Native, (5) Asian, (6) other, and (7) unknown. Among participating mothers, we found good agreement between self-reported ethnicity and ethnicity from birth records (white 96\%, black 95\%, Hispanic 98\%). To include our entire sample of eligible families for these analyses, we used ethnicity from birth records.

\section{Statistical analysis}

We tabulated frequencies of the major CHDs among eligible infants for each site separately and for the NDSP as a whole. We used simple $\chi^{2}$ analyses to examine the occurrence of each major CHD by site, ethnicity, sex, origin of the chromosome error, and maternal age group $(<35$ and $\geq 35$ ). We then calculated odds ratios (OR) for each major $\mathrm{CHD}$ by logistic regression using presence or absence of the defect as the dependent variable, ethnicity and sex as independent variables, and adjusting for maternal age at birth of the child and NDSP site.

\section{Laboratory studies}

Each site was responsible for obtaining blood or buccal samples on enrolled infants and their parents. Details on sample collection and processing as well as the methodology used for parent and stage of origin studies are available elsewhere. ${ }^{1}$

Supplementary analyses were performed to determine if the observed ethnic/racial differences may be explained, in part, by genetic factors. To do this, we used AIMs. AIMs are genetic loci with large differences in allele frequency between populations and can be used to infer individual geographic ancestry. ${ }^{15}$ Us- ing DNA samples from a subset of our infants whose parents self-identified as black, DNAPrint ${ }^{\circledR}$ (Sarasota, FL) genotyped a panel of 164 AIMs to estimate the admixture proportions of the four major population groups (African, European, East Asian, Native American) using maximum likelihood estimate analysis as described by Frudakis et al. ${ }^{16}$ Thirty-seven black infants with DS and complete AVSD (cases) and 37 black infants with DS and no CHD (controls) were tested. We included nine non-NDSP cases ascertained specifically because of having DS and a complete AVSD. One additional control was ascertained as part of a larger DS and CHD study. ${ }^{14} \mathrm{AIMs}$ on chromosome 21 (3 markers) were excluded from the analysis because the standard genotype scoring algorithm could not interpret trisomic genotype signals. On the recommendation of DNAPrint, we also excluded samples with 40 or more failed markers. Thirtyfour cases and 31 controls genotyped for 161 autosomal AIMS remained for the final analysis. We used the $t$ test to compare the proportions of African alleles in case and control samples.

\section{RESULTS}

The NDSP ascertained 1469 infants with DS among the six participating sites. At each site the expected number of infants based on the birth population of the covered area correlated well with the actual number of DS cases identified (Table 2). Overall, $74 \%$ of eligible families participated fully or partially (maternal questionnaire with or without buccal sample). The participation rates varied by site (AR 84\%, CA 65\%, GA 75\%, IA $77 \%$, NJ $81 \%$, NY 76\%). Cardiac information was based on echocardiograms, cardiac catheterization, or surgery in $88 \%$ of the cases (range by site 75-98\%).

\section{Cardiac defects}

One or more major cardiac defects were present in $44.2 \%$ of NDSP-eligible infants. Among all infants, the rates for AVSD, ASDII, and VSD were similar $(17.2 \%, 18.6 \%$, and $19.2 \%$, respectively) (Table 3 ). The type of VSD was not always specified, but among the 227 with that information, $65 \%$ were membranous and 35\% were muscular. Because only 39 infants $(2.7 \%)$ had TOF, that defect was not included in further analyses. In Table 4, we present AVSD frequencies two ways: (1) complete AVSD and (2) any AVSD. The latter includes complete, partial (AVSD-type ASD or VSD), and those for which the type of AVSD was not specified. We found no association between the presence of any CHD and gastrointestinal defects including esophageal atresia, tracheoesophageal fistula, duodenal atresia/stenosis, annular pancreas, Hirschsprung disease, or imperforate anus (data not shown).

\section{Origin of nondisjunction}

Of the 787 cases for which biological samples were available and the origin of the extra chromosome 21 could be determined, $93 \%$ of nondisjunction events were maternal meiotic errors ( $76 \%$ meiosis I, $24 \%$ meiosis II) and only $4 \%$ were paternal ( $42 \%$ meiosis I, 58\% meiosis II). Three percent were 
Table 2

National Down Syndrome Project: Down syndrome births—expected, identified, eligible; and cardiac diagnostic methods used

\begin{tabular}{|c|c|c|c|c|c|c|}
\hline Site/study period/birth years & Yearly births & $\begin{array}{c}\text { DS live } \\
\text { births } / 10,000\end{array}$ & $\begin{array}{l}\text { Expected trisomy } \\
21 \text { or mosaic } \\
\text { births }^{c}\end{array}$ & $\begin{array}{c}\text { Identified } \\
\text { (\% of expected) }\end{array}$ & Eligible $^{d}$ & $\begin{array}{l}N(\%) \text { with echocardiogram, } \\
\text { cardiac catheterization, or } \\
\text { surgery }\end{array}$ \\
\hline Arkansas statewide 10/00-9/03 & $37,000 \times 3 \mathrm{yrs}$ & $11.08^{a}$ & 118 & $111(94.1)$ & 96 & $79(82.3)$ \\
\hline California 3 counties $1 / 01-6 / 03$ & $186,000 \times 2.5 \mathrm{yrs}$ & $10.14^{a}$ & 453 & $544(120.1)$ & 501 & $377(75.2)$ \\
\hline $\begin{array}{l}\text { Georgia 5-county Atlanta area } \\
\text { 1/01-9/04 }\end{array}$ & $50,963 \times 3.75 \mathrm{yrs}$ & $12.49^{a}$ & 229 & $228(99.6)$ & 202 & $198(98.0)$ \\
\hline Iowa statewide 2001-2003 & $37,768 \times 3 \mathrm{yrs}$ & $13.97^{b}$ & 152 & $143(94.1)$ & 126 & $119(94.4)$ \\
\hline New Jersey statewide 1/01-6/04 & $115,745 \times 3.5 \mathrm{yrs}$ & $11.34^{a}$ & 441 & $480(108.84)$ & 395 & $373(94.4)$ \\
\hline New York 15 counties 10/00-9/03 & $47,256 \times 3 \mathrm{yrs}$ & $10.28^{a}$ & 140 & $167(119.3)$ & 149 & $145(96.7)$ \\
\hline Total & & & 1533 & $1673(109.1)$ & 1469 & $1291(87.9)$ \\
\hline
\end{tabular}

${ }^{a}$ Prevalence figures taken from National Birth Defects Prevention Network. ${ }^{17}$

${ }^{b}$ Unpublished data, Paul Romitti, Director, Iowa Registry for Congenital and Inherited Disorders.

${ }^{c}$ Total DS expected during study period minus $4 \%$ due to chromosome translocation.

${ }^{d}$ Eligibility criteria for present report: Mother spoke English or Spanish, child was not adopted or deceased, standard trisomy 21 without additional clinically important chromosome abnormality. Mosaics excluded.

Table 3

National Down Syndrome Project: major congenital heart defects

\begin{tabular}{|c|c|c|}
\hline & $N$ & $\%$ \\
\hline Atrioventricular septal defect & 252 & 17.2 \\
\hline Complete & 188 & \\
\hline Atrial component only & 19 & \\
\hline $\begin{array}{l}\text { Ventricular component } \\
\text { only }\end{array}$ & 31 & \\
\hline $\begin{array}{l}\text { Atrioventricular defect } \\
\text { NOS }\end{array}$ & 14 & \\
\hline Atrial septal defect ${ }^{a}$ & 273 & 18.6 \\
\hline Ventricular septal defect ${ }^{b}$ & 282 & 19.2 \\
\hline Membranous & 147 & \\
\hline Muscular & 80 & \\
\hline NOS & 55 & \\
\hline Tetralogy of Fallot & 39 & 2.7 \\
\hline Without AVSD & 29 & \\
\hline With AVSD & 10 & \\
\hline Other $^{c}$ & 19 & 1.3 \\
\hline \multicolumn{3}{|l|}{ Summary } \\
\hline Cases with $>1$ of the above & 649 & 44.2 \\
\hline Cases with none of the above & 820 & 55.8 \\
\hline Total & 1469 & \\
\hline
\end{tabular}

${ }^{a}$ Secundum ASD. Excludes PFO and PFO versus ASD.

${ }^{b}$ Excludes VSD that is part of an AVSD or TOF.

Includes double outlet right ventricle (6), coarctation of aorta (6), dextrocardia (2), right aortic arch (5).

NOS, not otherwise specified.

mitotic in origin. The presence or absence of specific heart defects or all CHDs combined did not vary by parent or stage of origin of nondisjunction.

\section{Maternal age}

Eligible mothers were equally divided between those younger than 35 years at delivery (50.5\%) and those 35 years or older $(49.5 \%)$. We did not find statistically significant differences between these two groups of women in the percentage of AVSD or ASDII in their offspring with DS (Table 4), but there were fewer VSDs among the infants born to women 35 years or older.

\section{Infant sex}

The sex ratio for all NDSP-eligible infants with DS was 1.15 (787 male, 682 female) and did not differ by ethnicity. When each CHD was examined separately, AVSD showed a significant difference between sexes with approximately twice as many female infants as male infants affected (Table 4). Among infants with AVSD, a preponderance of female infants was clearly evident in whites (35M:59F) and blacks (16M:29F), but not in Hispanics (20M:21F). There were too few Asians for an accurate comparison (2M:3F). Female infants had a small increased risk for ASDII (OR, 1.35; 95\% CI, 1.03-1.76).

\section{Maternal ethnicity}

Whites were represented at $\geq 10 \%$ at all six NDSP sites, five sites had $\geq 10 \%$ Hispanics, and three sites had $\geq 10 \%$ blacks. Significant ethnic differences in the prevalence of CHDs were apparent for AVSDs. Based on all eligible infants and using whites as the referent group, blacks with DS were twice as likely to be born with a complete AVSD (adjusted OR, 2.06; 95\% CI, 1.32-3.21), whereas Hispanics were one half as likely (adjusted OR, 0.48; 95\% CI, 0.30-0.77) (Table 4). Although the numbers were small, Asian infants also showed a trend toward fewer AVSDs. An increased risk for ASDII among black infants was marginally significant (OR, 1.63; 95\% CI, 1.06-2.50). There was good agreement among sites regarding these ethnic trends (data not shown). Using self-reported ethnicity from the maternal questionnaire did not significantly alter the odds 
Table 4

National Down Syndrome Project: major congenital heart defects by maternal age, infant sex, and maternal ethnicity

\begin{tabular}{|c|c|c|c|c|c|c|c|c|c|}
\hline & \multirow[b]{2}{*}{$N$} & \multicolumn{2}{|c|}{ Complete AVSD } & \multicolumn{2}{|c|}{ Any AVSD } & \multicolumn{2}{|r|}{ ASDII } & \multicolumn{2}{|r|}{ VSD } \\
\hline & & $\%^{a}$ & OR $(95 \% \mathrm{CI})^{b}$ & $\%^{a}$ & $0 \mathrm{R}(95 \% \mathrm{CI})^{b}$ & $\%^{a}$ & OR $(95 \% \mathrm{CI})^{b}$ & $\%^{a}$ & OR $(95 \% \mathrm{CI})^{b}$ \\
\hline \multicolumn{10}{|l|}{ Mother's age } \\
\hline$<35$ & 735 & 13.7 & ref & 18.2 & ref & 19.3 & ref & 21.4 & ref \\
\hline$>35$ & 721 & 12.1 & $0.85(0.62-1.17)$ & 16.2 & $0.86(0.66-1.16)$ & 18.2 & $0.95(0.73-1.25)$ & 17.2 & $0.76(0.58-0.99)$ \\
\hline Male & 787 & 9.5 & ref & 9.5 & ref & 16.5 & ref & 20.0 & ref \\
\hline Female & 682 & 16.6 & $1.93(1.40-2.67)$ & 16.6 & $2.06(1.55-2.75)$ & 21.0 & $1.35(1.03-1.76)$ & 19.1 & $0.95(0.73-1.24)$ \\
\hline \multicolumn{10}{|c|}{ Mother's race } \\
\hline White & 624 & 15.1 & ref & 19.2 & ref & 14.9 & ref & 17.1 & ref \\
\hline Black & 183 & 24.6 & $2.06(1.32-3.21)$ & 29.5 & $1.98(1.31-2.99)$ & 25.7 & $1.63(1.06-2.50)$ & 20.2 & $1.06(0.68-1.65)$ \\
\hline Hispanic & 569 & 7.2 & $0.48(0.30-0.77)$ & 11.6 & $0.60(0.40-0.99)$ & 20.9 & $1.23(0.85-1.79)$ & 22.5 & $1.23(0.87-1.76)$ \\
\hline Asian & 63 & 7.9 & $0.52(0.20-1.36)$ & 11.1 & $0.57(0.25-1.31)$ & 17.5 & $1.15(0.57-3.02)$ & 15.9 & $0.92(0.45-1.90)$ \\
\hline
\end{tabular}

${ }^{a}$ Percentage of infants of specified maternal age, sex, or ethnicity with the named heart defect.

${ }^{b}$ Logistic regression model included maternal age and ethnicity, infant sex, and site.

Complete AVSD, complete atrioventricular septal defect; any AVSD, complete, partial, and unspecified AVSD; ASDII, secundum atrial septal defect (excludes PFO or PFO versus ASD); VSD, ventricular septal defect (excludes AVSD-type VSD and VSD that is part of TOF).

ratios for the various heart defects. Further, when we removed the $10 \%$ of cases in which the mother reported that she and the father of the infant were of different ethnicities, there was no significant change in the CHD frequencies (data not shown). Comparing the frequencies of AVSD, ASDII, and VSD between enrolled and nonparticipating infants, we did not find any significant differences for any ethnic group (data not shown). We also determined that there were no differences between the ethnic groups in the proportion of families who became ineligible because their child died after birth.

Because diagnostic methods could affect the detection rate of CHDs, we examined the use of echocardiography, cardiac catheterization, and surgery among ethnic groups. For all sites combined, there was no significant difference in the use of these methodologies between whites and blacks (93\% whites, 92\% blacks), but significantly fewer of these procedures were reported among Hispanics (83\%). Because CA had a high proportion of Hispanics and reported an overall lower use of these diagnostic tools than the other sites, we examined the CA data separately and found $70 \%$ of whites and $77 \%$ of Hispanics were diagnosed by at least one of these methods. For all other sites combined, a similar percentage of whites (95\%) and Hispanics (93\%) had one or more of these procedures. Thus the overall lower rate of echocardiography among Hispanics likely was due to a high proportion of NDSP Hispanics being from CA, where the use of echocardiography among all ethnic groups was lowest.

To investigate further the role of ethnicity in the occurrence of AVSD, we stratified the NDSP sample by birth country of the mother and found significant differences in the percentage of infants born with AVSD to black and Hispanic mothers depending on whether the mother was born in the United States or elsewhere. Infants with DS born to black mothers born outside the United States, mainly in Africa and the Caribbean, had a higher percentage of AVSDs than did infants of
Table 5

Number (\%) of infants with AVSD by birth country of mother for whites, blacks, and Hispanics

\begin{tabular}{lcccccc}
\hline & & & \multicolumn{3}{c}{ Complete AVSD } \\
\cline { 5 - 6 } Ethnicity & Birth country & & $N(\%)^{a}$ & & $\%$ & $P$ \\
\hline \multirow{2}{*}{ White } & US & 485 & 72 & $14.9^{b}$ & NS \\
& Other & $27(5.3)$ & 3 & 11.1 & \\
\multirow{3}{*}{ Black } & US & & 91 & 18 & 19.8 & 0.036 \\
& Other & & $25(21.6)$ & 10 & 40.0 & \\
\multirow{2}{*}{ Hispanic } & US & & 73 & 10 & 13.7 & 0.022 \\
& Other & $335(82)$ & 20 & 6.0 & \\
\hline
\end{tabular}

${ }^{a}$ Enrolled families only.

${ }^{b}$ Interpretation: of white infants whose mothers were born in the US, $14.9 \%$ had an AVSD.

US, United States.

black mothers born in the United States. Infants of Hispanic mothers born outside the United States, mainly in Mexico and Central America, had fewer AVSDs than did infants of Hispanic mothers born inside the United States. We did not observe differences by birth country for whites (Table 5).

Because our earlier report describing CHDs in the ADSP population covered only the first 6 years of the 11-year study, ${ }^{2}$ we reexamined the full data set comprising Atlanta infants born between 1989 and 1999 (Table 1). We did not find a higher rate of AVSD in blacks compared with that in whites (16.1\% blacks, $N=182 ; 17.6 \%$ whites, $N=210)$. On further evaluation, we found that only $8.3 \%$ of blacks in the ADSP were born outside the United States compared with $21.6 \%$ overall in the NDSP. Among 26 ADSP-eligible Hispanics, only one had an AVSD (3.9\%), a low rate comparable to that found in the NDSP. The birth country of the mother was known for 


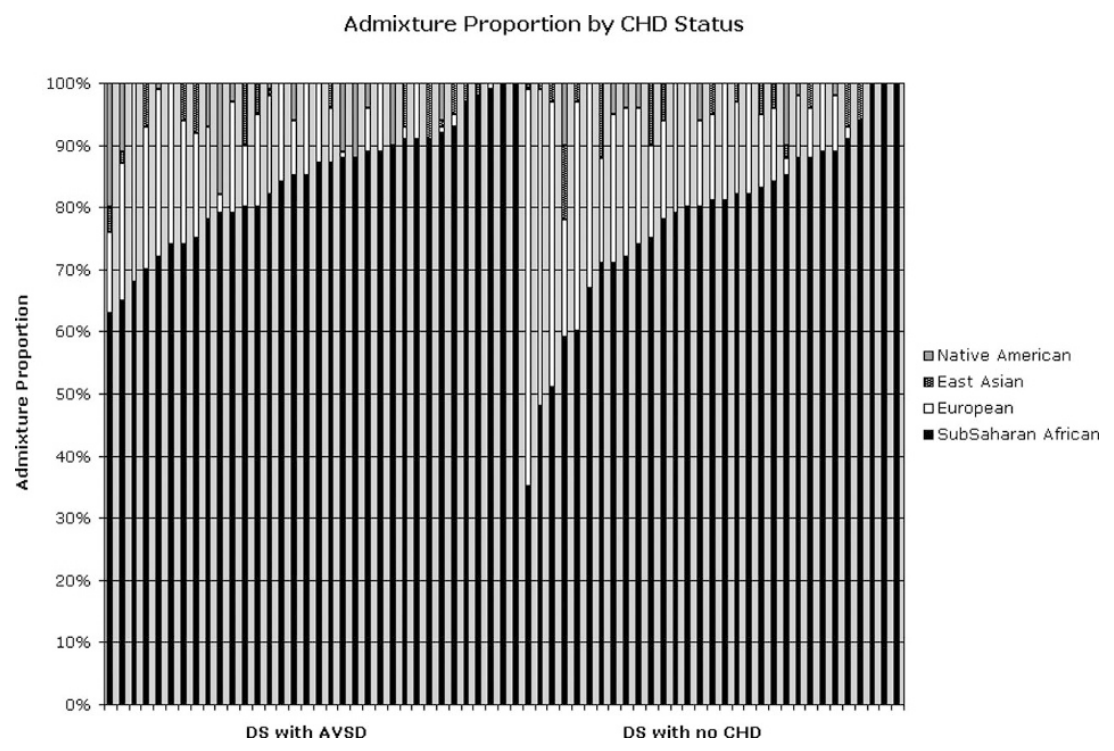

Fig. 1. A higher proportion of Sub-Saharan African (black) alleles was observed in cases (Down syndrome with complete atrioventricular septal defect), than in controls (Down syndrome with no congenital heart defects).

the 16 enrolled Hispanic ADSP families. All but one of these mothers was born outside of the United States.

\section{Assessment of ancestral information markers among black infants}

The higher incidence of AVSDs observed among NDSPeligible black infants, particularly among those whose mothers were born outside the United States, led us to hypothesize that genetic risk factors for AVSD may exist. To test this, we conducted a preliminary analysis among a subset of black infants to determine if those with AVSD had a higher proportion of ancestral African alleles compared with those with no heart defect. We used AIMs for this analysis. First, we found that Sub-Saharan African alleles made up the majority of alleles observed in the overall study sample of infants with self-identified black parents, as expected (Fig. 1). Consistent with our hypothesis, there was a significantly higher proportion $(P=$ 0.029) of Sub-Saharan African alleles among black infants with DS and AVSD (83.1\%), than in black infants with DS and no CHD (77.6\%) when compared by $t$ test.

\section{DISCUSSION}

The NDSP was designed to collect a unique combination of infant medical data, questionnaire responses from mothers, and DNA samples from the parents and child. The current report takes advantage of this exceptional data set as well as the diversity represented in this multisite sample to document the occurrence of CHDs in DS and explore relationships between DS-related CHDs and maternal age, ethnicity, infant sex, and the origin of the nondisjunction error.

We found similar proportions of DS infants with CHDs in the NDSP (44\%) and ADSP (41\%). Prevalence rates in other recent population-based studies have ranged from $23 \%$ to $56 \%$ (Table 1). Although most studies incorporate a figure for the overall proportion of heart defects, it is arguably more useful to report major CHDs separately to reduce the differences in rates due simply to the choice of defects included and to encourage an examination of the etiologies of the various defects. With approximately $66 \%$ of AVSD occurring in association with DS, ${ }^{9}$ this hallmark defect is of major interest.

In the NDSP, a partial or complete AVSD was present in $17 \%$ of eligible infants ( $39 \%$ of those with a reported CHD), a rate similar to that found in most other studies (Table 1 and $^{18-}$ 22). In contrast, ASDII rates varied widely among studies with the NDSP rate being the highest ${ }^{18-23}$ (Table 1) even though we excluded atrial defects described as PFO or PFO/ASDII. Although we do not have an explanation, similar rates at the six NDSP sites suggest our findings are a true representation of ASDII in DS. VSD rates in the population-based studies listed in Table 1 ranged from $11 \%$ to $44 \%$ of all CHDs. The predominance of perimembranous VSD over other types in the NDSP has been noted by others in individuals with and without DS.9,24 Interestingly, the 1998 California report found a VSD in only $11 \%$ of DS infants, whereas in the NDSP, California reported $22 \%$. This difference may be due in part to differences in the ethnic mix of the two populations. Compared with a subset of those earlier CA cases reported by Torfs and Christianson, ${ }^{10}$ the proportion of Hispanics in the NDSP appears to be approximately $10 \%$ higher. Although not significant, we found a trend toward higher VSD rates in NDSP Hispanics.

The lack of an association between maternal age and the frequency of AVSD or ASDII in infants with DS has been reported in previous studies. ${ }^{2,7,10}$ Further, our findings did not confirm the observation by Kallen et al. ${ }^{3}$ of fewer CHDs, especially AVSD and VSD, in teenage mothers. In seeking an explanation for the slightly lower rate of VSD in infants of older mothers, it may be important to consider the effect of prenatal testing. For example, pregnancies in older women may be 
monitored more closely by ultrasonography. Detection of a fetal heart defect may lead to amniocentesis, fetal karyotyping, and elective termination of DS fetuses affected with CHD. In this regard, both AVSD and ASDII also demonstrated lower odds ratios among older women although these values did not reach significance.

The predominance of female infants among those with AVSD has been reported previously in individuals with and without DS. ${ }^{3,9,25-27}$ Some studies have noted more female infants among those with DS and a VSD, ${ }^{3,25}$ whereas others including the current study have not. ${ }^{26}$ The small increase in ASDII among female infants could be real or, alternatively, could be the result of diagnostic misclassification among some NDSP infants in whom ASDs which were actually primary (ASDI) and typical of AVSD were classified as ASDII. Park et al. ${ }^{25}$ found no sex difference among those with an ASD.

The NDSP is the first population-based study of DS and CHDs to have three ethnic groups represented at a greater than $10 \%$ frequency (Table 1). This permitted a direct examination of possible differences in CHD rates among ethnic groups. AVSDs demonstrated the most striking ethnic differences. Specifically, black infants with DS had about twice the risk of AVSD as white infants, whereas Hispanics had one-half the risk of whites. Similar ethnic differences in AVSD rates at multiple sites strengthen the overall NDSP findings. In contrast, we found no significant ethnic differences in VSD rates in the NDSP as a whole or among the sites (data not shown). As noted earlier for female infants, diagnostic misclassification of an ASDI as an ASDII might provide an explanation for the observed increase in ASDII among blacks.

In exploring possible confounders that could account for the observed ethnic differences in AVSD rates, we have ruled out the ethnic disparities both in the use of modern diagnostic methods such as echocardiography and in the death rate of NDSP infants. Further, gestational age or birth weight could influence the length of hospitalization after birth and, in turn, might dictate the type of cardiac evaluation completed. However, we did not find any ethnic differences in mean gestational age (data not shown). Both blacks and Hispanics had a lower birth weight than did whites (data not shown) but, because black infants were more likely to have an AVSD than were whites, whereas Hispanics were less likely, birth weight did not seem to correlate with AVSD rates.

The fact that AVSD has traditionally been reported as the most common CHD among infants with DS in North American and European studies probably reflects the fact that the populations surveyed consisted largely of white and, to a lesser extent, black individuals with DS (Table 1). Black-versuswhite comparisons have rarely been made and the results have been conflicting. ${ }^{7,9}$

Although we found no previous population-based studies of CHDs among Hispanic infants with DS, Vida et al. ${ }^{13}$ found VSD to be the most common and AVSD the least common CHD among 349 Guatemalan infants presenting for a cardiac evaluation. Similarly, de Rubens Figueroa et al..$^{12}$ reported that VSD, ASD, and PDA were the most common defects in Mexi- can children with DS. Only 8\% were diagnosed with an AVSD; however, differential survival based on cardiac status may have been a factor because participating individuals ranged up to 13 years or age. In the United States, Torfs and Christianson ${ }^{10}$ reported that in CA the prevalence of AVSD appeared to be lower for Hispanics than for whites. These studies plus the current report document a lower rate of AVSD for Hispanics both in their native countries and among those who have immigrated to the United States. Arguably this points toward genetic rather than environmental factors having the major role.

Similar to the findings among Hispanics, VSD has been reported to be the most common CHD and AVSD the least common among Asian individuals with DS. $6,11,28$ Although the NDSP identified only 63 infants of Asian mothers, we noted that ASD and VSD were the most common CHD, whereas the AVSD rate (7.9\%) was similar to the Hispanic rate (7.2\%). The evolutionary relationship between Asian and Native American populations is well known, ${ }^{29,30}$ and varying degrees of Native American admixture have been demonstrated among Hispanic-American communities. $^{31}$

We conducted two post hoc analyses to test the hypothesis that genes may contribute to the risk for AVSDs among infants with DS and that such genes may explain some of the observed ethnic variation. To do this, we took advantage of the fact that the United States black population comprises recent immigrants from Africa and the Caribbean as well as a large admixed population of African Americans. ${ }^{32,33}$ It is well known that African Americans exhibit increased racial admixture compared with native Africans, ${ }^{34}$ and thus our observation that infants of black women born outside of the United States are more likely to have an AVSD than are infants of black mothers born inside the United States strengthens the idea that allelic differences among ethnic groups may play a role in the risk for AVSD. The fact that we did not see a similar increase in AVSDs in blacks in our ADSP may reflect the fact that the black population in that study was born largely in the United States.

The second set of data supporting a genetic contribution to the risk of AVSD comes from our preliminary analysis of AIMs among black infants with and without AVSD. The observed increased proportion of Sub-Saharan African allelic variants among the former group is consistent with a role for genes in abnormal heart development. More importantly, this difference suggests a strategy for gene discovery for AVSD using admixture linkage disequilibrium (MALD) ${ }^{35}$ The MALD approach takes advantage of long blocks of LD temporarily created by the mixing of two parental populations (in this case European and African) to identify genetic regions of the highrisk population that are preserved in the affected admixed. The heterogeneous US population is ideal for these types of studies.

Alternative explanations for the increased proportion of African alleles among black infants with AVSD could include chance because of small sample size. Clearly, additional work with ancestral markers is needed. As well, similar efforts should be made to understand the lower incidence of AVSD among Hispanics. Interestingly, infants of Hispanic mothers who immigrated to the United States had a lower risk for AVSD than 


\section{Freeman et al.}

did infants of Hispanic mothers born inside this country. It is well documented that US Hispanic communities represent various combinations of ancestral populations including European, Native American, and African. ${ }^{31}$ If the interpretation of the AIM data among African Americans is true, a higher rate of African alleles in Hispanic cases with DS and AVSD would suggest an ancient AVSD risk factor common to many populations. A higher proportion of alleles from other populations, Native American for example, might suggest a different, protective allele in the population. Most importantly, our preliminary data suggest that the time and effort required to ascertain a racially and culturally diverse population are worthwhile.

In summary, the strengths of the NDSP include its large size, population basis, and ethnic diversity. Because recruitment occurred nationally at six locations, observations and trends could be compared among sites. Further, the NDSP collected medical information on infants, questionnaire responses from their mothers, and biological samples from the parents and child. As evident from the current report, this combined data set constitutes a major resource in efforts to understand the etiology of CHDs in DS. Limitations of the study include the fact that only families in which the mother spoke English or Spanish were eligible. In addition, we were not able to include pregnancy losses, terminations, stillbirths, or infants who died after birth but before the family could be enrolled.

The NDSP demonstrates that the diversity of the US population is a valuable asset to epidemiological studies of genetic and environmental influences on DS and its associated phenotype. In future studies, we will continue to use this data set to explore the mechanisms underlying the observed link between ethnicity and CHDs.

\section{ACKNOWLEDGMENTS}

This work was supported by NIH R01 HD38979, NIH P01 HD24605, F32 HD046337, Children's Health Care of Atlanta Cardiac Research Committee and by the technical assistance of the General Clinical Research Center at Emory University (NIH/NCRR M01 RR00039). We thank the many families nationwide whose participation has made this study possible. In addition, we thank all the personnel at each NDSP site.

\section{References}

1. Freeman SB, Allen EG, Oxford-Wright CL, Tinker SW, et al. The National Down Syndrome Project: design and implementation. Public Health Rep 2007;122:62-72.

2. Freeman SB, Taft LF, Dooley KJ, Allran K, et al. Population-based study of congenital heart defects in Down syndrome. [see comment]. Am J Med Genet 1998;80:213217.

3. Kallen B, Mastroiacovo P, Robert E. Major congenital malformations in Down syndrome. Am J Med Genet 1996;65:160-166.

4. Stoll C, Alembik Y, Dott B, Roth MP. Study of Down syndrome in 238,942 consecutive births. Ann Genet 1998;41:44-51.

5. Torfs CP, Christianson RE. Anomalies in Down syndrome individuals in a large population-based registry. Am J Med Genet 1998;77:431-438.

6. Lo NS, Leung PM, Lau KC, Yeung CY. Congenital cardiovascular malformations in Chinese children with Down's syndrome. Chin Med J (Engl) 1989;102:382-386.

7. Khoury MJ, Erickson JD. Improved ascertainment of cardiovascular malformations in infants with Down's syndrome, Atlanta, 1968 through 1989. Implications for the interpretation of increasing rates of cardiovascular malformations in surveillance systems. Am J Epidemiol 1992;136:1457-1464.

8. Marino B. Patterns of congenital heart disease and associated cardiac anomalies in children with Down syndrome. In: Marino B, Pueschel SM, eds. Heart disease in persons with Down syndrome. Baltimore: Paul Brookes, 1996:133-140.

9. Ferencz C, Loffredo CA, Correa-Villasenor A, Wilson PD, eds. Genetic and environmental risk factors of major cardiovascular malformations: the BaltimoreWashington Infant Study: 1981-1989. Armonk, NY: Futura, 1997. Perspectives in Pediatric Cardiology; No. 5.

10. Torfs CP, Christianson RE. Maternal risk factors and major associated defects in infants with Down syndrome. Epidemiology 1999;10:264-270.

11. Jacobs EG, Leung MP, Karlberg J. Distribution of symptomatic congenital heart disease in Hong Kong. Pediatr Cardiol 2000;21:148-157.

12. de Rubens Figueroa J, del Pozzo Magana B, Pablos Hach JL, Calderon Jimenez C, et al. [Heart malformations in children with Down syndrome]. Rev Esp Cardiol 2003; 56:894-899.

13. Vida VL, Barnoya J, Larrazabal LA, Gaitan G, et al. Congenital cardiac disease in children with Down's syndrome in Guatemala. Cardiol Young 2005;15:286-290.

14. Kerstann KF, Feingold E, Freeman SB, Bean LJ, et al. Linkage disequilibrium mapping in trisomic populations: analytical approaches and an application to congenital heart defects in Down syndrome. Genet Epidemiol 2004;27:240-251.

15. Shriver MD, Smith MW, Jin L, Marcini A, et al. Ethnic-affiliation estimation by use of population-specific DNA markers. Am J Hum Genet 1997;60:957-964.

16. Frudakis TN, Thomas MJ, Ginjupali SN, Handelin B, et al. CYP2D6 4 polymorphism is associated with statin-induced muscle effects. Pharmacogenet Genomics 2007;17:695-707.

17. Birth defects surveillance data from selected states, 1996-2000. Birth Defects Res A Clin Mol Teratol 2003;67:729-818.

18. Rowe RD, Uchida IA. Cardiac malformation in mongolism. Am J Med 1961;31:726735 .

19. Wells GL, Barker SE, Finley SC, Colvin EV, et al. Congenital heart disease in infants with Down's syndrome. South Med J 1994;87:724-727.

20. Fixler DE, Threlkeld N. Prenatal exposures and congenital heart defects in Down syndrome infants. Teratology 1998;58:6-12.

21. Frid C, Drott P, Lundell B, Rasmussen F, et al. Mortality in Down's syndrome in relation to congenital malformations. J Intellect Disabil Res 1999;43(Pt 3):234-241.

22. Calzolari E, Garani G, Cocchi G, Magnani C, et al. Congenital heart defects: 15 years of experience of the Emilia-Romagna Registry (Italy). Eur J Epidemiol 2003;18:773780.

23. Spahis JK, Wilson GN. Down syndrome: perinatal complications and counseling experiences in 216 patients. Am J Med Genet 1999;89:96-99.

24. Marino B, Papa M, Guccione P, Corno A, et al. Ventricular septal defect in Down syndrome. Anatomic types and associated malformations. Am J Dis Child 1990;144: $544-545$.

25. Park SC, Mathews RA, Zuberbuhler JR, Rowe RD, et al. Down syndrome with congenital heart malformation. Am J Dis Child 1977;131:29-33.

26. Pinto F, Nunes L, Ferraz F, Sampayo F. Down's syndrome: different distribution of congenital heart diseases between the sexes. Int J Cardiol 1990;27:175-178.

27. Harris JA, Francannet C, Pradat P, Robert E. The epidemiology of cardiovascular defects. II. A study based on data from three large registries of congenital malformations. Pediatr Cardiol 2003;24:222-235.

28. Matsuo N, Oshima M, Naganuma M, Shimizu K, et al. Major and minor anomalies in Japanese children with Down's syndrome. Jpn Heart J 1972;13:307-316.

29. Horai S, Kondo R, Nakagawa-Hattori Y, Hayashi S, et al. Peopling of the Americas, founded by four major lineages of mitochondrial DNA. Mol Biol Evol 1993;10:2347.

30. Nei M, Roychoudhury AK. Evolutionary relationships of human populations on a global scale. Mol Biol Evol 1993;10:927-943.

31. Bertoni B, Budowle B, Sans M, Barton SA, et al. Admixture in Hispanics: distribution of ancestral population contributions in the Continental United States. Hum Biol 2003;75:1-11.

32. Parra EJ, Marcini A, Akey J, Martinson J, et al. Estimating African American admixture proportions by use of population-specific alleles. Am J Hum Genet 1998;63: 1839-1851.

33. Parra EJ, Kittles RA, Argyropoulos G, Pfaff CL, et al. Ancestral proportions and admixture dynamics in geographically defined African Americans living in South Carolina. Am J Phys Anthropol 2001;114:18-29.

34. McKeigue PM, Carpenter JR, Parra EJ, Shriver MD. Estimation of admixture and detection of linkage in admixed populations by a Bayesian approach: application to African-American populations. Ann Hum Genet 2000;64:171-186.

35. Smith MW, O'Brien SJ. Mapping by admixture linkage disequilibrium: advances, limitations and guidelines. Nat Rev Genet 2005;6:623-632. 